\section{Decentralising HIV treatment in lower- and middle-income countries}

\author{
Tamara Kredo, Nathan Ford, Folasade B. Adeniyi, Paul Garner \\ The independent commentary was written by Juvencio \\ Furtado
}

\begin{abstract}
BACKGROUND: Policy makers, health staff and communities recognise that health services in lower- and middle-income countries need to improve people's access to HIV treatment and retention to treatment programmes. One strategy is to move antiretroviral delivery from hospitals to more peripheral health facilities or even beyond health facilities. This could increase the number of people with access to care, improve health outcomes, and enhance retention in treatment programmes. On the other hand, providing care at less sophisticated levels in the health service or at community-level may decrease quality of care and result in worse health outcomes. To address these uncertainties, we summarised the research studies examining the risks and benefits of decentralising antiretroviral therapy service delivery.
\end{abstract}

OBJECTIVES: To assess the effects of various models that decentralised HIV treatment and care to more basic levels in the health system for initiating and maintaining antiretroviral therapy.

METHODS:

Search methods: We conducted a comprehensive search to identify all relevant studies regardless of language or publication status (published, unpublished, in press, and in progress) from 1 January 1996 to 31 March 2013, and contacted relevant organisations and researchers. The search terms included "decentralisation", "down referral", "delivery of health care", and "health services accessibility".

Selection criteria: Our inclusion criteria were controlled trials (randomised and non-randomised), controlled-before and after studies, and cohorts (prospective and retrospective) in which HIV-infected people were either initiated on antiretroviral therapy or maintained on therapy in a decentralised setting in lower- and middle-income countries. We define decentralisation as providing treatment at a more basic level in the health system to the comparator.

Data collection and analysis: Two authors applied the inclusion criteria and extracted data independently. We designed a framework to describe different decentralisation strategies, and then grouped studies against these strategies. Data were pooled using random-effects metaanalysis. Because loss to follow up in HIV programmes is known to include some deaths, we used attrition as our primary outcome, defined as death plus loss to follow-up. We assessed evidence quality with GRADE methodology.

MAIN RESULTS: Sixteen studies met the inclusion criteria, all but one were from Africa, comprising two cluster randomised trials and 14 cohort studies. Antiretroviral therapy started at a hospital and maintained at a health centre (partial decentralisation) probably reduces attrition (RR $0.46,95 \% \mathrm{Cl} 0.29$ to $0.71,4$ studies, 39090 patients, moderate quality evidence). There may be fewer patients lost to care with this model (RR $0.55,95 \%$ Cl 0.45 to 0.69 , low quality evidence).

We are uncertain whether there is a difference in attrition for antiretroviral therapy started and maintained at a health centre (full decentralisa- tion) compared to a hospital at 12 months (RR $0.70,95 \% \mathrm{Cl} 0.47$ to 1.02 four studies, 56360 patients, very low quality evidence), but there are probably fewer patients lost to care with this model (RR 0.3, 95\% Cl 0.17 to 0.54 , moderate quality evidence).

When antiretroviral maintenance therapy is delivered at home by trained volunteers, there is probably no difference in attrition at 12 months (RR 0.95, 95\% Cl 0.62 to 1.46, two trials, 1453 patients, moderate quality evidence).

AUTHORS' CONCLUSIONS: Decentralisation of HIV care aims to improve patient access and retention in care. Most data were from good quality cohort studies but confounding between site of treatment and outcomes cannot be excluded. Nevertheless, this review found that attrition appears to be lower in partial decentralisation models of treatment, where antiretrovirals were started at hospital and continued in the health centre; with antiretroviral drugs started and continued at health centres, no difference in attrition was detected, but there were fewer patients lost to care. For antiretroviral therapy provided at home by trained volunteers, no difference in outcomes were detected when compared to facility-based care.

This is the abstract of a Cochrane Review published in the Cochrane Database of Systematic Reviews (CDSR) 2013, issue 6. Art. No.: CD009987. DOI: 10.1002/14651858.CD009987.pub2 (http://onlinelibrary.wiley.com/doi/10.1002/14651858.CD009987.pub2/abstract). For full citation and authors' details, see reference 1 .

The full text of this review is freely available from: http://onlinelibrary. wiley.com/doi/10.1002/14651858.CD009987.pub2/pdf.

\section{REFERENCE}

1. Kredo T, Ford N, Adeniyi FB, Garner P. Decentralising HIV treatment in lower- and middle-income countries. Cochrane Database Syst Rev 2013;6:CD009987.

\section{COMMENTS}

Decentralization and simplification of healthcare for patients with HIV/AIDS has been the subject of discussion and controversy around the world, especially in developing countries, as this systematic review indicates.

Making decisions in this regard needs to be based on the particular features of each country. In Brazil, through the "universalization" of HIV/AIDS treatment, there has been an attempt to adopt this type of service as standard. In the same way as at global level, such decisions need to obey specific criteria. In Brazil, because of its continental characteristics and its cultural and socioeconomic diversity, evaluations in this regard should also be conducted.

In areas in which medical assistance is precarious, either due to a lack of well-trained healthcare professionals or due to complete absence of these professionals, including physicians, this type of care should certainly be implemented, even if provisionally.

Monitoring and treating of people living with HIV/AIDS in Brazil has reached a quality level that, even though not ideal, needs to be maintained. It is unreasonable to backtrack in this process, in the name of cost reduction, when such costs have not yet been proven.

Juvencio Furtado. Professor of Infectious Diseases, Faculdade de Medicina do ABC (FMABC), and Head of the Department of Infectious Diseases at Hospital Heliópolis, São Paulo, Brazil. 